\title{
Breve acercamiento a la mediación cultural: hacia una delimitación del campo de estudio y una aproximación a sus aplicaciones didácticas en la combinación de lenguas español-italiano
}

\author{
Giuseppe TROVATO \\ Università degli Studi di Catania (Italia) \\ Departamento de Ciencias de la Formación \\ univcatania@gmail.com
}

Recibido: octubre 2012

Aceptado: enero 2013

\begin{abstract}
RESUMEN
La presente aportación tiene como objetivo analizar el concepto de mediación cultural, noción que ha empezado a cobrar importancia a raíz de los numerosos flujos migratorios que la mayoría de los países occidentales han venido experimentando a partir de los años 90 del siglo pasado. Nuestro propósito reside en enmarcar la cuestión desde el punto de vista de la investigación científica en la materia $\mathrm{y}$, a continuación, nos proponemos ofrecer algunas reflexiones relacionadas con el perfil del mediador cultural y con los contextos en los que esta figura profesional desarrolla su labor de mediación. En última instancia, plantearemos una situación comunicativa concreta, esto es, un "guión" que se puede explotar a efectos didácticos en las clases de mediación lingüístico-cultural españolitaliano.

Palabras clave: Mediación cultural, flujos migratorios, dimensiones de la mediación, perfil del mediador cultural, situación comunicativa.

Brève étude de la médiation culturelle : vers une délimitation du champ d'étude y quelques applications didactiques relatives à la combinaison

\section{RÉSUMÉ} linguistique espagnol-italien
\end{abstract}

Cette contribution a pour but d'analyser le concept de médiation culturelle, notion qui est devenue de plus en plus importante dans le sillage des nombreux flux migratoires que la plupart des pays occidentaux ont connus depuis les années 90 du siècle dernier. Notre objectif est ainsi celui d'examiner avant tout la problématique du point de vue de la recherche scientifique sur la matière, et ensuite, celui d'offrir quelques réflexions liées au profil du médiateur culturel et aux contextes dans lesquels cette figure professionnelle développe ses activités de médiation. Pour terminer, nous allons présenter une situation de communication spécifique, c'est à dire un "script" qui pourrait être exploité dans le domaine de la didactique de la médiation linguistique et culturelle entre l'espagnol et l'italien.

Mots-clé: Médiation culturelle, flux migratoires, dimensions de la médiation, profil du médiateur culturel, situation de communication. 
Studying Cultural Mediation: towards a definition of the field of study and didactic applications for the Spanish-Italian language combination

\begin{abstract}
In this paper, the concept of cultural mediation, a notion that has increasingly gained momentum in the wake of the migratory flows in most Western countries since the last decade of the past century is analyzed. A scientific approach to researching into this set of issues is our main purpose. Based on our research outcome, a few guidelines on what we deem might be the professional profile and context in which a cultural mediator works are suggested. In the final analysis, a Spanish-Italian communication case-study template which we feel can be useful to teachers of cultural linguistic mediation courses is discussed.
\end{abstract}

Keywords: Cultural Mediation, migratory flows, dimensions of Mediation, cultural mediator profile, script.

SUMARIO: 0 . Introducción. 1. La Mediación cultural: delimitación de un campo de estudio complejo. 2. Origen y desarrollo de la mediación cultural. 3. ¿Qué significa concretamente la mediación cultural? 4. Las dimensiones de la mediación cultural. 5. El mediador cultural: perfil y características. 6. La didáctica de la mediación cultural: algunas observaciones sobre el par de lenguas español-italiano. 6.1. Guión de la situación comunicativa: la mediación cultural en el ámbito comunitario. 6.2. Algunos comentarios sobre el guión propuesto: análisis de los problemas contrastivos español-italiano. 7. Reflexiones conclusivas. 8. Referencias Bibliográficas.

\title{
0. INTRODUCCIÓN
}

La comunicación entre los seres humanos ha desempeñado un papel paradigmático desde la noche de los tiempos, pues los hombres - al entrar en contacto - han advertido la necesidad de establecer relaciones personales, sociales y económicas:

La necesidad del ser humano de comunicarse, la sensación de vivir en un modo cada vez más uniforme y la existencia de vías de comunicación cada vez más eficaces y rápidas nos impide con frecuencia ver otras realidades cercanas en las que la comunicación no es tan fácil ni tan efectiva ni cuenta con las mismas herramientas para llevarla a cabo.

(Valero Garcés, 2010: XI)

Al hilo de esta cita, no cabe duda de que la comunicación siempre ha sido la tónica en las relaciones entre los hombres, aun manifestándose dificultades en términos lingüísticos, culturales y pragmáticos. Dichas dificultades, a lo largo de los siglos, se han hecho cada vez más patentes y contundentes, especialmente en el ámbito cultural, lo que ha provocado durante mucho tiempo conflictos y guerras con un único denominador común: la ausencia de canales de comunicación 
eficaces, esto es, la falta de entendimiento entre individuos o grupos culturales distintos.

Hoy en día, es muy frecuente oír hablar de "mediación" y "mediador" cultural, a pesar de que se trata de conceptos aún en vías de consolidación, tanto en el plano de la formación como en el ámbito laboral. Es indudable que no es tarea fácil alcanzar una definición unánime en torno al concepto de mediación cultural ${ }^{1}$, pues varios investigadores y académicos han intentado examinar a fondo la cuestión y encontrar terreno común para afianzar su papel en términos de investigación científica. Asimismo, no se puede pasar por alto la distinción entre mediación lingüística (ML) y MC, objeto de análisis y debate en la producción científica de numerosos estudiosos ${ }^{2}$.

\section{LA MEDIACIÓN CULTURAL: DELIMITACIÓN DE UN CAMPO DE ESTUDIO COMPLEJO}

El tema de la MC se tiene que abordar, en nuestro criterio, desde un punto de vista multidisciplinar, es decir, tomando en consideración varios enfoques y perspectivas así como la aproximación a distintas disciplinas ${ }^{3}$. En efecto, mediar es sinónimo de agilizar la comunicación entre dos partes que, por varias razones, no logran entenderse tal y como lo harían dos personas pertenecientes a la misma cultura. En este sentido, la mediación asume un papel muy similar al de la traducción oral o interpretación ${ }^{4}$, ya que entran en juego los aspectos lingüísticos típicos de la oralidad, cuya complejidad no se puede infravalorar en el marco de la comunicación mediada.

Sin embargo, al entrar en contacto dos individuos o dos grupos de personas, la vertiente puramente lingüística sólo representa la "cima del iceberg", por así decirlo. No es nuestra intención subestimar la función que cumple la lengua en el transcurso de la comunicación mediada por una tercera parte, pues somos conscientes de que la transmisión de un mensaje pasa, ante todo, a través de un canal fónico. De ahí que todos los ítems léxicos, semánticos y morfosintácticos jueguen un papel sumamente relevante en el flujo de las informaciones. Así y todo,

${ }^{1}$ A partir de ahora MC. Al referirnos a la mediación lingüística y cultural, usaremos MLC.

${ }^{2}$ No vamos a deternernos en el tema, ya que se ha abordado con más exhaustividad en otros trabajos. A este respecto, consúltense, entre otros, Blini (2009); Carreras i Goicoechea \& Pérez Vázquez (eds.) (2010); De Luise \& Morelli (eds.) (2012); González Rodríguez (2006; 2010); Rudvin (2005); Russo \& Mack (eds.) (2005).

${ }^{3}$ Consúltese, a este propósito, una aportación nuestra (2012), donde abordamos la MC en el ámbito sanitario.

${ }^{4}$ Para una disertación más profundizada sobre el papel del mediador como intérprete, véase Morelli (2010). La autora lleva a cabo un análisis pormenorizado y claro sobre el parentesco que existe entre disciplinas como la mediación, la traducción y la interpretación. 
en este estudio nos planteamos una reflexión - si cabe puntual - sobre la influencia que ejerce el componente cultural en el ámbito de la mediación.

Para ejemplificar lo susodicho y adentrarnos más directamente en el tema que nos ocupa, nos parece conveniente mencionar lo que Raga Gimeno y Sales Salvador (2010: 166) plantean con respecto a la mediación (inter)cultural:

\begin{abstract}
Partamos de una evidencia difícilmente cuestionable: cuando una persona de origen chino, pakistaní, senegalés o marroquí acude a los servicios sanitarios españoles, o europeos en general, corre el riesgo de encontrarse con problemas lingüísticos y con problemas culturales; y aunque todos estemos de acuerdo en que los primeros presentan mayor urgencia, no es menos cierto que los problemas culturales pueden en ocasiones provocar problemas comunicativos con consecuencias gravísimas, cuando no dramáticas.
\end{abstract}

Estas consideraciones, aparentemente sencillas y descontadas, nos proporcionan elementos útiles para seguir analizando el concepto de MC y definir su ámbito de aplicación.

Ahora bien, la problemática intercultural se manifiesta en todo tipo de comunicación o interacción donde entren en contacto dos individuos que interpretan la realidad según su propia idiosincrasia, que adoptan posturas y actitudes diferentes, en función de un determinado contexto comunicativo y reaccionan ante una situación con arreglo a determinadas pautas culturales, típicas del entorno en el que se han formado. Este es, a grandes rasgos, el marco en el que se sitúa la MC, pues al chocar las pautas culturales antes aludidas, se produce un conflicto o bien una situación marcada por tensión y ansiedad. Tanto es así, que con el fin de salvar las dificultades que puedan surgir a raíz de las diferencias culturales, se hace necesaria una figura profesional específica y especializada, cuyo objetivo se materializa en acortar las distancias entre las partes involucradas y hacer que se llegue a un entendimiento mutuo: el mediador cultural.

\title{
2. ORIGEN Y DESARROLLO DE LA MC
}

Antes de esbozar el perfil del mediador cultural, es conveniente profundizar en el significado de la $\mathrm{MC}$ y ver cómo se ha desarrollado. Ahora bien, no es tarea fácil detectar el origen exacto de la MC, ya que se trata de un fenómeno social e histórico que siempre ha acompañado a los hombres en el momento de poner en marcha contactos con sus similares. Sin detenernos en un análisis pormenorizado sobre el origen de la MC, nos parece útil acudir a los planteamientos de Fernández Sánchez (2001: 1), quien centra su interés en el origen de la interpretación ${ }^{5}$ :

\footnotetext{
${ }^{5}$ A pesar de que las reflexiones ofrecidas por la autora se refieren a la interpretación, nosotros mantenemos que su introducción histórica se adapta muy acertadamente al ámbito de la MC.
} 
La investigación historiográfica se apoya en documentos. La historiografía de la interpretación no es una excepción, aunque la escasez de fuentes, por lo menos, hasta el siglo XX, y su carácter desigual y disperso dificultan evidentemente la tarea del investigador.

A continuación, la autora pone de relieve que (ibidem):

La existencia y modalidades de la traducción oral - como las de otras prácticas orales, las traducciones invisibles de las que habla Gargatagli (1997) por ejemplo, esos mecanismos de transmisión oral mediante los cuales numerosos mitos y leyendas se transmitieron de una cultura a otra - no siempre han quedado reflejadas en la cultura escrita, lo que no le resta importancia, más bien al contrario, su invisibilidad nos remonta a épocas antiquísimas, a vastos espacios territoriales. Concretamente a las antiguas civilizaciones imperiales del Próximo Oriente por lo que respecta a la cultura occidental, dando cuenta de la amplitud y de la importancia de los contactos culturales a lo largo de los siglos así como de la ineludible necesidad humana de comunicarse ${ }^{6}$.

Una vez más nos percatamos del papel relevante que desempeña la comunicación en las relaciones entre individuos, los cuales nunca han escatimado esfuerzos en aras de comprender a los demás, franquear obstáculos lingüísticos y culturales y, en definitiva, establecer relaciones sólidas y pacíficas. Siguiendo a Fernández Sánchez (op. cit.: 5), es posible demostrar que:

La historia documentada de la interpretación se remonta al tercer milenio a.C., en la necrópolis que contiene los monumentos funerarios de los príncipes de Elefantina, en las fronteras de Egipto con Sudán. En las inscripciones de estos monumentos encontramos las primeras referencias acerca del uso de intérpretes ${ }^{7}$ en las misiones comerciales y diplomáticas llevadas a cabo por estos altos dignatarios, los príncipes de Elefantina, entre los faraones de la $6^{\text {a }}$ dinastía (2340 a.C.) y la antigua población africana de los nubios.

La autora ofrece una visión panorámica muy esclarecedora, efectuando un recorrido a través de los siglos. Sin duda alguna, uno de los momentos más emblemáticos que han marcado la evolución de la mediación en su sentido más amplio, viene representado por la época de los descubrimientos donde «los abundantes ejemplos de malentendidos y de fallos en la comunicación [...] nos remiten con toda su crudeza a la profunda diferencia conceptual entre las culturas implicadas» (Fernández Sánchez, 2010: 12).

\footnotetext{
${ }^{6}$ La negrita es nuestra.

${ }^{7}$ Nosotros diríamos "mediadores".
} 
A lo largo de los siglos, los contactos entre individuos y comunidades lingüísticas se han ido intensificando gracias a los intercambios comerciales, políticos y culturales y gracias también a la creación de nuevos canales de comunicación y al desarrollo de la tecnología. Ahora bien, el fenómeno de la globalización ha conllevado, sin lugar a dudas, cambios sustanciales en la forma de vivir e interactuar con los demás y de enfrentarse a "lo otro". Centrándose específicamente en la situación que la MC ha venido experimentando en Italia en las últimas décadas, Valdiviezo Valdivieso (2010: 237) observa que:

[...] il bisogno di un servizio di mediazione culturale arriva agli inizi degli anni '90, momento in cui si assiste a un mutamento della fase del ciclo migratorio dei cittadini stranieri residenti nel territorio, vale a dire, da una prima fase, in cui ci si concentrava nell'inserimento dei singoli, siamo passati alla seconda fase, dove si punta alla stabilizzazione ed integrazione dei nuclei familiari di cittadini immigrati, che passano ad ottenere una condizione di minoranza etnica.

Parece evidente, pues, que los fenómenos migratorios han marcado una nueva configuración de la vida en sociedad, considerada como una aldea global, por hablar en términos de consecuencias socioculturales acarreadas por la globalización. Efectivamente, es innegable que hoy día vivimos en entornos multilingües y pluriculturales, pues los medios de comunicación nos han permitido conocer "la otredad" y saber interpretar correctamente determinados gestos o convenciones culturales, característicos de un determinado pueblo ${ }^{8}$. A modo de ejemplo, la forma en que los italianos usamos el cuerpo o la voz para expresar un determinado mensaje no se corresponde necesariamente, ni mucho menos, con las modalidades adoptadas por los chinos o los rusos, a saber, culturas muy lejanas. Otro tanto puede decirse, sin embargo, de culturas menos distantes, como la española o latinoamericana. Es un craso error pensar que la afinidad lingüística es sinónimo de afinidad cultural. Basta con dirigir la mirada hacia la cercana España: cualquier italiano que haya ido a España, de vacaciones o por motivos de estudio, se habrá dado cuenta de que a la hora de conocer y saludar a alguien, las cosas varían con respecto a como actuamos los italianos. En realidad, los italianos besamos antes la mejilla izquierda y luego la derecha y, por lo general, estrechamos las manos. En España, en cambio, se empieza por la derecha y luego se pasa a la izquierda y no se da la costumbre de estrechar la mano. Los aspectos en los que acabamos de incidir no se pueden menospreciar a lo largo de la interacción con nuestro(s) interlocutor(es) extranjero(s), pues suponen una aproximación a una cultura "otra", digna de la misma consideración que la nuestra. A modo de

${ }^{8}$ Cf. Geertz (1973) para un análisis más exhaustivo sobre el tema de la interpretación de culturas diferentes. 
ilustración y de cierre de este apartado, proponemos un extracto de un artículo de Antonio Cattarossi ${ }^{9}$, sobre cómo se besan los españoles:

Recuerdo que, recién llegado a España, noté una diferencia de costumbres a la que hay que tener cuidado para evitar meter la pata. En Italia, cuando nos presentan a una persona (que sea hombre o mujer da igual), solemos estrecharle la mano y decir: "Piacere". En España, en cambio, cuando uno se presenta a una mujer las manos no se utilizan para nada, sino que le da directamente dos besos a la vez que dice: "Encantado" o: "Mucho gusto" (obviamente no estoy hablando de encuentros formales o reuniones de trabajo donde es normal estrecharse la mano). Parece una tontería pero no es para nada inusual ver situaciones embarazosas donde una chica española alarga el cuello mientras que el chico italiano extiende la mano (quedando como un empanado). Y no es todo. Una vez aclarado como hay que portarse cuando nos presentan a una chica hay que tener cuidado con otra diferencia más: los dos besos italianos, en las situaciones en las que se utilizan, son distintos de los españoles!!!

\section{3. ¿QUÉ SIGNIFICA CONCRETAMENTE LA MC?}

En el panorama actual, marcado por la globalización y la libre circulación de seres humanos, la MC adquiere una función indispensable, orientada a agilizar el proceso de integración de los inmigrantes dentro de nuestras fronteras. Asimismo, se plantea que la sociedad de acogida esté preparada ante los cambios interculturales que se producen a raíz de la interacción entre dos culturas distintas. La MC se perfila, pues, como un puente entre dos partes, favoreciendo así el conocimiento mutuo de culturas, valores, tradiciones y sistemas sociales, en una óptica de intercambio y enriquecimiento mutuos. Además, es interesante considerar la MC como parte integrante de las políticas de integración social, pues permite que los ciudadanos extranjeros gocen de sus derechos fundamentales y que nuestras sociedades se abran a la "otredad". En líneas generales, las que resumimos a continuación son las finalidades del proceso de MC:

- Franquear las barreras culturales que obstaculizan la comunicación entre los inmigrantes y los servicios e instituciones del país de acogida.

- Agilizar la realización de todos los trámites burocráticos orientados hacia la integración de los ciudadanos extranjeros y extracomunitarios.

- Mejorar y adecuar la calidad de los servicios que la sociedad de acogida ofrece a estas personas.

- Promover la incorporación al mercado laboral de la población inmigrada así como su inserción en el ámbito escolar, cultural y sociosanitario.

9 A continuación ofrecemos el enlace del que hemos extraído el texto propuesto: http://www.antoniocattarossi.com/blog/es/2007/06/11/presentaciones-y-besos/. 
- Desarrollar una labor de mediación social, con el fin de eludir situaciones conflictivas entre las comunidades recién llegadas y las instituciones locales.

- Emprender actividades de prevención y superación de conflictos y organizar cursos de formación y capacitación para que las nuevas comunidades se familiaricen con el nuevo entorno social y cultural.

\section{LAS DIMENSIONES DE LA MC}

$\mathrm{Al}$ abordar la $\mathrm{MC}$, es importante - a nuestro juicio - ahondar en que se trata de un complejo proceso de descodificación de la comunicación, a lo largo del cual se pueden apreciar tres dimensiones. En otras palabras, la actividad mediadora se despliega en función de tres niveles de análisis. A continuación, ofrecemos una ejemplificación de lo anteriormente dicho:

- Dimensión facilitadora: esta dimensión engloba el conjunto de tareas, funciones y operaciones que el mediador lleva a cabo, con el objetivo de facilitar la integración de las personas para las que desarrolla su labor de mediación. De modo que el mediador traduce a nivel lingüístico y pragmático, ofrece información sobre la sociedad de acogida y los trámites burocráticos que hay que realizar para hacerse con el permiso de residencia, asistir a la escuela o encontrar un trabajo, por mencionar algunos ejemplos. Así pues, cumple una importante función de intermediación entre el ciudadano extranjero y el servicio social ${ }^{10}$, con miras a que se produzca un acercamiento, en términos culturales y de comportamientos. Planteemos la siguiente situación: estamos en un hospital y tenemos que mediar un encuentro entre una mujer musulmana y un médico varón. En nuestra calidad de mediadores, nos corresponderá explicar, tanto a la mujer como al facultativo, cuáles son las respectivas pautas culturales y comportamentales, con el fin de sortear toda situación problemática que pueda surgir en el transcurso de la interacción.

- Dimensión comunicativa: la dimensión comunicativa de la MC está relacionada con todos los aspectos lingüísticos de la comunicación mediada. De modo que la mediación adquiere las características de la traducción oral o interpretación $\mathrm{y}$, desde este punto de vista, sirve para evitar malentendidos y superar bloques comunicativos. De hecho, al mediador no le corresponde sólo traducir fielmente mensajes e informaciones, sino que tiene que aclarar cualquier aspecto implícito de la comunicación, sobre todo en el terreno pragmático y cultural.

\footnotetext{
${ }^{10}$ Con "servicio social" hacemos referencia a varias instituciones, donde se hace necesaria la labor de MC: estructuras hospitalarias, jefaturas de policía, tribunales, centros de inmigrantes, centros escolares, oficinas de empleo, por mencionar las más comunes.
} 
- Dimensión psico-social: quizás sea la dimensión más caracterizadora de la MC. Se trata, efectivamente, de establecer una relación de confianza y de empatía entre los servicios del país de acogida y los ciudadanos extranjeros. En este marco, el mediador cultural desempeña un papel muy delicado, puesto que tiene que favorecer las instancias de ambas partes y actuar de manera objetiva. Por lo tanto, la imparcialidad ha de orientar su actuación como puente y facilitador de la comunicación, además de jugar un papel muy activo y dinámico en la promoción y difusión de nuevos valores y hábitos, a saber, los de las comunidades de inmigrantes. Lo anterior puede redundar en beneficio de la integración, en su sentido más amplio. En definitiva, es en esta dimensión donde la MC encuentra su máxima expresión y se configura como agente de cambio social y responsable del enriquecimiento cultural.

\section{EL MEDIADOR CULTURAL: PERFIL Y CARACTERÍSTICAS}

Tras estas notas introductorias que - dicho sea de paso - no se han planteado un análisis exhaustivo sobre el tema, puesto que afrontar este campo de estudio sería una apuesta que no nos atrevemos a emprender en este artículo, por razones de espacio.

En el párrafo dos, introdujimos la figura del mediador cultural. En este apartado, vamos a ahondar en el tema, buscando definir el perfil de esta figura puente, cuyo papel se desarrolla en varios ámbitos profesionales y socioculturales y con colectivos de personas heterogéneos.

Para poner en marcha nuestro análisis sobre el perfil del mediador cultural, consideramos oportuno hacer hincapié en lo que afirma Luka (2005: 203):

Il tratto fondamentale del mediatore è senza dubbio l'appartenenza ad una cultura "altra" che entra in relazione con la cultura maggioritaria della società di accoglienza, unita alla capacità di far interagire i due sistemi, valorizzando i punti di forza di entrambi e creando un sistema di reciprocità e di scambio tra le diverse regole di vita e di organizzazione sociale.

No es fácil encontrar un acuerdo sobre el papel y los rasgos distintivos de esta figura profesional que - a la altura del año 2013 - sigue sin contar con un reconocimiento oficial ${ }^{11}$. Probablemente el hecho de que en términos profesionales esta figura no goce de una identidad propia hace el panorama sombrío. Aun así, no queremos entrar en más detalles sobre la cuestión y alabamos los esfuerzos realizados por algunas asociaciones de categoría que operan en el contexto italiano,

${ }^{11}$ Es suficiente con considerar que en Italia no existe un Colegio de Mediadores. 
para que se reconozcan figuras profesionales como los mediadores lingüísticos y culturales $^{12}$.

Volviendo a las características más representativas del mediador cultural, Luka (ibídem) pone de relieve un aspecto emblemático:

Un altro tratto distintivo del mediatore culturale è dato dal conoscere profondamente la lingua e la cultura degli immigrati, in quanto lui stesso "immigrato" dal paese di provenienza dei destinatari della mediazione. Questa comunanza lo "avvicina" ai suoi utenti ed è il presupposto per una comprensione profonda dei loro problemi, oltre a rappresentare un grande potenziale di risposta.

Por consiguiente, el hecho de haber experimentado la misma condición de inmigrante, hace que el mediador sea especialmente sensible ante determinadas cuestiones relacionadas con su integración en la sociedad de acogida, su incorporación al mundo laboral y su interacción con la comunidad presente en el territorio. El mediador se presenta, pues, como un operador social que conoce bien, tanto la lengua y cultura de la comunidad minoritaria como las de la comunidad receptora a la vez que cuenta con competencias y destrezas específicas que le permiten interpretar correctamente las exigencias de las personas para las que lleva a cabo su actividad de mediación. A modo de resumen, enumeramos los requisitos que tiene que reunir un buen mediador cultural para ofrecer un servicio de calidad:

- Ser preferentemente de origen extranjero y hacer vivido anteriormente la condición de inmigrante.

- Conocer en profundidad la cultura y la realidad social y económica del país de origen.

- Contar con sólidos conocimientos de la lengua y la cultura del país de acogida, tanto a nivel escrito como oral, habida cuenta de todos los elementos que intervienen en el flujo de la comunicación y que amenazan con enturbiarla si no se afrontan con eficacia y prontitud.

- Estar muy familiarizado con todos los servicios sociales, administrativos y burocráticos que el país de acogida pone a disposición de las comunidades inmigrantes, con vistas a su integración.

- Estar en posesión de una titulación académica ${ }^{13}$.

${ }^{12}$ En concreto, hacemos referencia a la asociación AITI (Asociación italiana de Traductores e Intérpretes) [http://www.aiti.org/] que está desempeñando un papel muy activo, planteando la cuestión ante el Parlamento italiano.

${ }^{13}$ La cuestión del título de estudio plantea muchos problemas en términos de formación. Nosotros mantenemos que para ejercer como mediador lingüístico-cultural es necesario haber conseguido un título expedido por una institución académica. No obstante, la realidad a la que nos enfrentamos en el día a día es distinta. Por lo general, quienes efectúan labores 
- Estar motivado y dispuesto a trabajar en contextos problemáticos, donde sea necesario mantener un elevado nivel de paciencia y empatía comunicativa, sin ningún tipo de prejuicios.

- Haber adquirido previamente experiencias específicas y concretas en el terreno de la MC.

Las que hemos venido planteando se pueden considerar, a grandes rasgos, como las características fundamentales del mediador. El único aspecto en el que quisiéramos centrar la atención estriba en la formación del mediador. Como comentábamos anteriormente, somos partidarios de que el mediador cuente con un título universitario, lo que le permitiría tener un mayor reconocimiento en el concierto de las profesiones. En efecto, hoy día, en el contexto académico italiano, existen cursos de grado en "Mediación lingüística e intercultural"14 , que expiden un título universitario de primer ciclo. Sin embargo, la formación de los mediadores lingüísticos y/o culturales viene desarrollada por entidades e instituciones públicas o privadas, financiadas con fondos procedentes generalmente de organizaciones como la Unión europea o las Regiones. El recorrido de formación consta, por lo general, de módulos teórico-metodológicos $\mathrm{y}$, sucesivamente, se desarrolla un período de prácticas en el marco de estructuras públicas donde los futuros mediadores entran en contacto con la realidad de la mediación y ponen en acción los conocimientos adquiridos en las clases teóricas.

\section{LA DIDÁCTICA DE LA MC: ALGUNAS OBSERVACIONES SOBRE EL PAR DE LENGUAS ESPAÑOL-ITALIANO}

Para finalizar nuestras reflexiones acerca de la MC, hemos decidido ofrecer algunos planteamientos didácticos, para traducir el marco teórico en la práctica. Ciertamente, abordar la didáctica de la MC significa adentrarse en un escenario complejo, en el que intervienen numerosos factores relacionados con la combinación de lenguas implicada, el contexto educativo en el que se lleva a cabo la didáctica, los destinatarios del curso, etc. Queremos aclarar que las orientaciones didácticas que ofrecemos tienen que ver con el contexto universitario. Para ser más concretos, se trata de una unidad de aprendizaje que se puede realizar en el marco

de $\mathrm{MC}$ son personas que cuentan con un título de nivel escolar $\mathrm{y}$, en algunos casos, ni siquiera ése.

${ }^{14}$ Se trata de licenciaturas de primer ciclo en "Ciencias de la Mediación lingüística" (Classe delle lauree in Scienze della Mediazione Linguistica L12). Para tener una visión más completa, remitimos al lector al enlace siguiente que da cuenta de la situación académica italiana sobre la cuestión: http://www.cestor.it/atenei/1012.htm. 
de las clases de $\mathrm{MLC}^{15}$. A continuación y a modo de ejemplo, brindamos de forma esquemática la ficha de la actividad propuesta ${ }^{16}$ :

\section{FICHA DE LA ACTIVIDAD DIDÁCTICA PROPUESTA}

1. Objetivos

$\checkmark$ Fomentar la competencia lingüística e intercultural de los estudiantes, acercándoles a una cultura distinta a la suya;

$\checkmark$ Desarrollar las técnicas específicas de la mediación oral y de la interpretación bilateral ${ }^{17}$;

$\checkmark$ Favorecer la competencia mediadora, a través de un situación comunicativa ad hoc;

$\checkmark$ Desarrollar la comunicación intercultural en el aula de Mediación a través del aprendizaje cooperativo ${ }^{18}$;

$\checkmark$ Impulsar la reflexión metalingüística y contrastiva sobre dos lenguas afines como el español y el italiano.

2. Combinación de lenguas: Español/italiano

3. Temas y contenidos abordados: Integración de inmigrantes en el país de acogida desde el punto de vista social y laboral

4. Nivel especifico recomendado: B2 del Marco Común Europeo de Referencia

5. Temporalización: 1 sesión de dos horas aproximadamente

${ }^{15}$ Cabe señalar que la actividad propuesta se coloca específicamente en el marco de las clases de técnicas de MLC oral, en el ámbito de una licenciatura de primer ciclo en mediación lingüística y se centra en el par de lenguas español-italiano. La combinación lingüística planteada se justifica por ser el sector científico-disciplinar en el que operamos (L-LIN/07 Lengua y Traducción - Lengua Española), según las directrices del MIUR (Ministerio de Educación italiano).

${ }^{16}$ La ficha didáctica propuesta no tiene ánimo de exhaustividad. Sólo se plantea como una guía para docentes de MLC que trabajen con el par de lenguas español-italiano. Claro está que cada profesor podrá concebir nuestra ficha a modo de orientación y adaptarla a los temas, contenidos y niveles que considere más apropiados.

${ }^{17}$ Remitimos al lector a un artículo nuestro (2011) donde abordamos la interpretación bilateral en relación con el par de lenguas español-italiano.

${ }^{18}$ Véase Bazzocchi (2006). 
6. Dinámica: individual / en parejas

7. Soportes para la didáctica: material entregado por el docente e información extraída de Internet (páginas web, sitios de organizaciones no gubernamentales, organizaciones internacionales que se ocupan del tema de la inmigración, documentación online recabada de las consejerías, ayuntamientos y jefaturas de policía).

\subsection{Guión de la situación comunicativa: la MC en el ámbito comunitario ${ }^{19}$}

A continuación, proponemos la situación comunicativa en la que estarán involucrados nuestros estudiantes de mediación español/italiano. Sin embargo, antes es oportuno proporcionar una contextualización del marco comunicativo abordado:

Un ciudadano peruano, que ha llegado a Italia hace muy poco, acude a una oficina de empleo para recibir información sobre los pasos que hay que dar para conseguir un trabajo en el país de acogida. La comunicación se desarrolla con la ayuda de un mediador cultural, ya que el ciudadano extracomunitario todavía no tiene conocimientos ni de la lengua italiana ni de su sistema burocrático y laboral. El mediador cultural cumplirá, pues, una función de enlace entre el hombre y el funcionario de la oficina de empleo.

\section{GUIÓN DE LA SITUACIÓN COMUNICATIVA}

A. Hola, buenos días. Me llamo Pedro Álvarez Durán, tengo 42 años y recién he llegado a Italia con mi familia para darles a mis hijos mejores posibilidades en su vida personal y profesional.

B. Buongiorno. Intanto mi dica... Da dove viene e cosa desidera specificamente?

A. Vengo de Perú y he venido a esta oficina para apuntarme a las listas de la oficina de empleo. Lo que pasa es que no sé qué documentos necesito, ya que no conozco el sistema italiano.

B. Guardi. Io non sono la persona preposta a dare questo tipo di informazioni.

${ }^{19}$ Consúltese Hale (2010) para una visión más completa sobre la mediación/interpretación en el ámbito comunitario. 
All'entrata, c'è un apposito 'information point' adibito a dare tutte le informazioni relative alla documentazione che deve presentare per potersi iscrivere all'ufficio di collocamento. Magari ritorni quando avrà tutti i documenti.

A. Mire, usted tiene razón, es que me encuentro en un país nuevo, apenas entiendo el italiano y la verdad es que he leido palabras que no consigo entender. ¿Qué es la "anagrafe" o la "questura", por ejemplo?

B. Allora, l'anagrafe è un ufficio comunale in cui, all'interno di un apposito registro, si documenta il numero e lo stato civile della popolazione residente sul territorio italiano. La questura, invece, è un ufficio dell'amministrazione statale che svolge compiti di pubblica sicurezza e rilascia i permessi di soggiorno ai cittadini stranieri.

A. Efectivamente todavía yo no tengo la residencia en Italia. Mi familia y yo nos alojamos en casa de unos amigos que viven en Italia desde hace ya mucho tiempo.

B. Ascolti, se vuole avere un posto di lavoro qui in Italia, deve necessariamente ottenere la residenza. Dopodiché, dovrà presentare anche altri documenti che attestino la sua formazione in ambito professionale e fornirci un suo curriculum vitae.

A. Hablando con otros compañeros peruanos, me he enterado de que los títulos y diplomas conseguidos en mi país no están reconocidos en Italia, porque no formamos parte de la Unión europea. La verdad es que yo no tengo una licenciatura, sino un título de perito comercial.

B. A me dispiace per la sua situazione ma non so come aiutarla in questo senso, perché il riconoscimento e la convalida dei titoli sono questioni di competenza del MIUR.

A. Sí, sí, ya lo sé. No quería achacarle a usted ninguna responsabilidad. Es que tengo que cuidar de mi familia y el hecho de no tener un trabajo me desmorona.

B. Guardi, come ha potuto notare, presso la nostra struttura ci sono dei mediatori culturali il cui compito è quello di guidare i cittadini stranieri ed extracomunitari nel loro percorso di orientamento e integrazione nel nostro 
paese. Loro potranno darle un quadro chiaro del funzionamento burocratico e amministrativo dell'Italia. Oltretutto parlano correntemente la sua lingua, quindi le assicuro che è in ottime mani.

A. Bueno, muchas gracias por sus consejos y espero hacerme con toda la documentación necesaria lo antes posible.

B. In bocca al lupo e l'aspetto prossimamente per sbrigare le sue pratiche.

\subsection{Algunos comentarios sobre el guión propuesto: análisis de algunos problemas contrastivos español-italiano}

La situación comunicativa presentada se enmarca en el ámbito social y comunitario y se presta muy bien a las simulaciones de técnicas de MLC españolitaliano. En primer lugar, cabe aclarar que hemos intentado oralizar el texto para agilizar la labor de intermediación de los estudiantes. A este propósito, lo ideal sería contar con la presencia, tanto del docente titular de la asignatura como del lector o auxiliar de conversación nativo, para que los estudiantes consideren la situación comunicativa lo más auténtica posible ${ }^{20}$. Además, es importante subrayar que la situación planteada es un claro ejemplo de MLC, puesto que el estudiante y ¿por qué no? - futuro profesional de la mediación, tendrá que efectuar ante todo una transposición lingüística, vehiculando mensajes concretos. Naturalmente, la mediación lingüística conlleva, en mayor o menor medida, una mediación entre culturas. En el caso que nos ocupa, es verdad que no estamos abordando dos culturas que están en las antípodas, como podrían ser la japonesa o la árabe. Sin embargo, es oportuno que nunca se infravaloren aspectos típicos de la comunicación intercultural, incluso al afrontar dos culturas aparentemente cercanas, pero lo suficientemente diferentes como para provocar equívocos y confusión.

Desde un punto de vista lingüístico, el fragmento no presenta especiales dificultades interpretativas o morfosintácticas para un estudiante italohablante. Una persona que conozca ambos idiomas, se habrá dado cuenta de que el nivel de lengua usado por el hombre extracomunitario es sensiblemente inferior con respecto al utilizado por el funcionario italiano, quien - como es de esperar - habla con mayor propiedad y conocimiento sobre el tema. Un aspecto que merece ser destacado es el uso de "recién he llegado", marcado diatópicamente como propio

\footnotetext{
${ }^{20}$ El guión propuesto forma parte de un corpus de situaciones simuladas que utilizamos para nuestras clases de interpretación bilateral español-italiano en el ámbito de una licenciatura trienal en "Teorías y técnicas de la Mediación lingüística". Nuestro propósito reside en ofrecer una fundamentación teórico-práctica sobre la dimensión intercultural de la comunicación.
} 
del habla de América Latina. Este uso es muy poco frecuente en el español peninsular. Por lo tanto, una primera conclusión que podemos plantear es que un mediador que tenga el español en su combinación lingüística no podrá prescindir de contar con sólidos conocimientos de las variedades diatópicas y, en ocasiones, diastráticas del español, sobre todo en el plano de la oralidad. En términos léxicos, el mediador tendrá que estar familiarizado con la terminología burocrática específica y la relacionada concretamente con el contexto italiano (ufficio di collocamento, anagrafe, questura, MIUR). Se trata de palabras que siempre estarán presentes en una interacción de este tipo, por lo que el mediador ha de reaccionar con prontitud y precisión ante la traducción de términos y locuciones característicos del sistema administrativo del país de acogida, pues es precisamente esta la información más solicitada por parte de los ciudadanos extranjeros. Si bien es cierto que el funcionario ofrece la definición de los conceptos de 'anagrafe' y 'questura', es deseable que el mediador haya automatizado la traducción y sea capaz de reproducir la explicación con exactitud y sin rodeos.

Aparte de la preparación terminológica, otro aspecto que consideramos especialmente relavante en la formación del mediador, reside en su preparación psicológica $^{21}$, ya que esta etapa «si pone l'obiettivo di aiutare lo studente a gestire la propria emotività e a non lasciarsi travolgere dall'ansia che può influire pesantemente sulla concentrazione» (Cotta-Ramusino, 2005: 58). También cabe señalar que el mediador operará a menudo en contextos de estrés y tensión, debido a la problemática intercultural y a las distancias que los interlocutores tienden a marcar entre sí. Asumido lo anterior, el profesional tendrá que haber desarrollado destrezas ad hoc, para no dejarse influenciar por factores ajenos.

Un último punto que nos gustaría traer a colación tiene que ver con aspectos como el tono de la voz y la gestualidad. Se trata de un conjunto de elementos ante los que un mediador no puede hacer la vista gorda, pues proporcionan una pista útil para la correcta interpretación de las intenciones y sentimientos de los destinatarios de la mediación. Probablemente el tono de la voz no deje mucho espacio para interpretaciones personales: cualquiera es capaz de detectar sentimientos como la ansiedad, la tensión, la alegría, la tristeza, la rabia o el miedo. Estas consideraciones, sin embargo, no las podemos aplicar tajantemente al ámbito de la gestualidad, pues los gestos conforman un lenguaje aparte y propio de una determinada idiosincrasia. La forma en que realizamos ciertas acciones como por ejemplo, mover las partes del cuerpo, sentarnos o sonreír está vinculada a la cultura en la que hemos crecido y nos hemos desarrollado. Al fin y al cabo, un mediador que se precie de tal, tendrá que prestar especial atención a este fenómeno y mostrar apertura y respeto ante formas de expresión física diferentes a la suya. Lo que

${ }^{21}$ Para una disertación más completa acerca de la didáctica de la mediación oral y la competencia comunicativa, véanse Cotta-Ramusino (2005) y Kaunzner (2005). 
acabamos de exponer no se puede aprender a través de libros o manuales, sino que son la práctica profesional y el contacto constante con los interlocutores los métodos más idóneos para desarrollar esta competencia.

\section{REFLEXIONES CONCLUSIVAS}

En este artículo nos hemos propuesto ofrecer un acercamiento a las bases de la MC, por tratarse de un campo de estudio complejo que se puede investigar desde numerosas perspectivas. Nuestro punto de partida ha sido enmarcar la cuestión bajo un perfil teórico: tras delimitar el ámbito de estudio y analizar el desarrollo de la disciplina, hemos examinado más a fondo sus finalidades y sus dimensiones, a saber, la facilitadora, la comunicativa y la psico-social. A continuación, hemos tratado de definir el perfil de quien desarrolla la labor de mediación, esto es, el mediador cultural, figura que también lleva a cabo una intermediación de tipo lingǘstico. De hecho no podemos olvidar que el binomio lengua / cultura representa la clave de la mediación (González Rodríguez, 2006: 251), pues se trata de dos aspectos estrechamente relacionados.

La última parte de nuestro estudio se ha planteado una reflexión de orden didáctico, más concretamente, cómo abordar la $\mathrm{MC}$ en el marco de lenguas afines: español-italiano. Para nuestros propósitos, hemos elaborado una ficha didáctica a través de la cual hemos explicitado los objetivos y los contenidos desarrollados, aclarando que tan sólo se propone como una herramienta de orientación en el amplio escenario de la didáctica de la mediación. Después, hemos presentado una situación comunicativa concreta que puede servir como "guión" para la formación de futuros mediadores lingüísticos y culturales y, seguidamente, hemos ofrecido algunos comentarios en torno a los problemas que la MC podría plantear aun trabajando con dos lenguas y culturas próximas ${ }^{22}$.

En conclusión, nuestra aportación ha perseguido aglutinar la vertiente teórica con la práctica, con el único objetivo de poner de manifiesto la versatilidad de la materia tratada así como el hecho de que para llevar a cabo una didáctica eficaz de la mediación, es imposible prescindir de sólidos fundamentos teóricos. Asimismo abogamos por que la formación en MLC se realice desde las instituciones académicas, con el fin de que la profesión goce de mayor prestigio y relevancia. Por último, y para retomar las palabras de dos estudiosas que se han ocupado de MLC, nuestro afán por la disciplina en cuestión ha venido motivado por «la necesidad de aclarar algunos puntos con respecto al rumbo que está tomando esta nueva materia en las carreras universitarias italianas y españolas», y brindar «a los profesionales de la mediación, que a menudo echan en falta, materiales que logren acercar la teoría a la práctica» (Carreras i Goicoechea, M. \& Pérez Vázquez, E., 2010: 20).

${ }^{22}$ No nos hemos extendido en los comentarios por motivos de espacio. 


\section{REFERENCIAS BIBLIOGRÁFICAS}

BAZZOCCHI, Gloria (2006): "El desarrollo de la comunicación intercultural en el aula de Mediación lingüística a través del aprendizaje cooperativo", en Bazzocchi, G. \& Capanaga, P. (eds.) (2006): Mediación lingüistica de lenguas afines: español/italiano, Bolonia, Gedit, 75-102.

BAZZOCCHI, Gloria \& CAPANAGA, Pilar (eds.) (2006): Mediación lingüistica de lenguas afines, español/italiano, Bolonia, Gedit.

BLINI, Lorenzo (2009): "La mediación lingüística en España e Italia: difusión de un concepto problemático", en ENTRECULTURAS, 1, 45-60, Disponible en: http://www.entreculturas.uma.es/n1pdf/articulo03.pdf [consulta: 28/01/2013].

CARRERAS I GOICOECHEA, Maria \& PÉREZ VÁZQUEZ, Enriqueta (eds.) (2010): "Introducción". en La mediación lingüistica y cultural y su didáctica, Bolonia, Bononia University Press, 11-21.

COTTA-RAMUSINO, Luisa (2005): "La mediazione linguistica orale tra didattica e professione", en RUSSO, Mariachiara y MACK, Gabriele (eds.) (2005): Interpretazione di Trattativa. La mediazione linguistico-culturale nel contesto formativo e professionale, Milán, Hoepli, 55-60.

DE LUISE, Danilo \& MORELLI, Mara (eds.) (2012): La mediazione comunitaria: un'esperienza possibile, Tricase, Libelluna Edizioni.

FERNÁNDEZ SÁNCHEZ, María Manuela (2001): "La práctica de la interpretación: introducción histórica", en COLLADOS AÍS, Ángela \& FERNÁNDEZ SÁNCHEZ, María Manuela (coords.) (2001): Manual de interpretación bilateral, Granada, Comares, 1-37.

GEERTZ, Clifford (1973) [1995]. La interpretación de las culturas, [Traducción de A. Bixio] Barcelona, Gedisa.

GONZÁLEZ RODRÍGUEZ, María Jesús (2006): "De lo escrito a lo oral y viceversa: una forma de ordenar el desorden en mediación", en BAZZOCCHI, Gloria \& CAPANAGA Pilar (eds.) (2006): Mediación lingüistica de lenguas afines: español/italiano, Bolonia, Gedit, 245-271.

GONZÁLEZ RODRÍGUEZ, María Jesús (2010): "El 'nuevo mundo' de la mediación lingüística y cultural: a la 'reconquista' del territorio desde la interpretación bilateral”, en CARRERAS I GOICOECHEA, Maria \& PÉREZ VÁZQUEZ, Enriqueta (eds.) (2010): La mediación lingüistica y cultural y su didáctica, Bolonia, Bononia University Press, 63-91.

HALE, Sandra Beatriz (2010): La Interpretación Comunitaria. La interpretación en los sectores jurídico, sanitario y social, (traducido por Rosa Cobas Álvarez y Carmen Valero Garcés), Granada, Editorial Comares.

KAUNZNER, Ulrike A (2005): "La competenza comunicativa come prerequisito della mediazione linguistica: proposte per una didattica preparatoria", en RUSSO, Mariachiara \& MACK, Gabriele (eds.) (2005): Interpretazione di Trattativa. La mediazione linguistico-culturale nel contesto formativo e professionale, Milán, Hoepli, 61-75. 
LUKA, Gino (2005): "Il mediatore interculturale", en RUSSO, Mariachiara \& MACK, Gabriele (eds.) (2005): Interpretazione di Trattativa. La mediazione linguistico-culturale nel contesto formativo e professionale, Milán, Hoepli, 203212.

MORELLI, Mara (2010): "Reflexiones sobre el papel del intérprete como mediador", en CARRERAS I GOICOECHEA, Maria \& PÉREZ VÁZQUEZ, Enriqueta (eds.) (2010): La mediación lingüistica y cultural y su didáctica, Bolonia, Bononia University Press, 93-110.

RAGA GIMENO, Francisco y SALES SALVADOR, Dora (2010): "El componente cultural en la formación para la interpretación y la mediación intercultural en el ámbito sanitario", en CARRERAS I GOICOECHEA, Maria \& PÉREZ VÁZQUEZ, Enriqueta (eds.) (2010): La mediación lingüística y cultural y su didáctica, Bolonia, Bononia University Press, 165-192.

RUDVIN, Mette (2005): "La formazione di interpreti in ambito sociale in Italia e all'estero", en RUSSO, Mariachiara \& MACK, Gabriele (eds.) (2005): Interpretazione di Trattativa. La mediazione linguistico-culturale nel contesto formativo e professionale, Milán, Hoepli, 131-143.

RUSSO, Mariachiara \& MACK, Gabriele (eds.) (2005): Interpretazione di Trattativa. La mediazione linguistico-culturale nel contesto formativo $e$ professionale, Milán, Hoepli.

SIEBETCHEU YOUMBI, Raymond (2012): "Identità e ruolo del mediatore linguistico-culturale in Italia", en The Journal of Cultural Mediation 1, [Amelia Burns y Francesco Caserta (eds.)], 13-30.

TROVATO, Giuseppe (2011): "La interpretación bilateral: algunas reflexiones metodológicas en torno a la combinación lingüística español-italiano", en redELE (Red Electrónica de didáctica del Español como Lengua Extranjera), 23. Disponible en: http://www.mecd.gob.es/redele/revistaRedEle/2011/ tercera.html [Consulta: 06/02/2013]

TROVATO, Giuseppe (2012): "La mediación (inter)cultural en el ámbito sanitario: algunas reflexiones relativas a las problemáticas culturales y al papel del mediador", en Redit (Revista Electrónica de Didáctica de la Traducción y la Interpretación), 8.

VALDIVIEZO VALDIVIESO, Anabel (2010): "Le esigenze formative dei mediatori culturali", en CARRERAS I GOICOECHEA, Maria \& PÉREZ VÁZQUEZ, Enriqueta (eds.) (2010): La mediación lingüística y cultural y su didáctica, Bolonia, Bononia University Press, 235-258.

VALERO GARCÉS, Carmen (ed.) (2005): Traducción como Mediación entre lenguas y culturas / Translation as Mediation or How to Bridge Linguistic and Cultural Gaps, Universidad de Alcalá, Servicios de Publicaciones.

VALERO GARCÉS, Carmen (2008) (segunda edición): Formas de mediación intercultural. Traducción e Interpretación en los servicios públicos, Granada, Editorial Comares. 
VALERO GARCÉS, Carmen (2010): "Prólogo del Editor". En HALE, Sandra Beatriz (2010): La Interpretación Comunitaria. La interpretación en los sectores jurídico, sanitario y social. (traducido por Rosa Cobas Álvarez y Carmen Valero Garcés), Granada, Editorial Comares, XI.

WADENSJÖ, Cecilia (1998): Interpreting as interaction, London and New York, Longman.

\section{SITOGRAFÍA}

http://www.antoniocattarossi.com/blog/es/2007/06/11/presentaciones-y-besos/ [consulta: 04/02/13] 